\title{
Posterior Cortical Atrophy: Review of the Recent Literature
}

\author{
François-Xavier Borruat
}

Published online: 18 October 2013

(C) Springer Science+Business Media New York 2013

\begin{abstract}
Posterior cortical atrophy (PCA) is a group of neurodegenerative dementing disorders characterized by initial predominant visual complaints followed by progressive decline in cognitive functions. The visuospatial and visuoperceptual defects arise from the dysfunction of, respectively, the dorsal (occipito-parietal) and the ventral (occipito-temporal) streams. Clinical symptoms, results of neuropsychological examination, and findings of posterior cerebral atrophy and/or posterior hypoperfusion/hypometabolism contribute to the diagnosis. However, owing to the insidious onset of PCA and the nonspecificity of initial symptoms, the diagnosis is often delayed. Specific etiologies include Alzheimer's disease, dementia with Lewy bodies, subcortical gliosis, corticobasal degeneration, and prion-associated diseases. Alzheimer's disease accounts for at least $80 \%$ of PCA cases. Recent research has concentrated on better defining the clinical presentation of PCA, improving neuroimaging analysis, testing new neuroimaging techniques, and developing biological measurements. Selected recent papers on PCA are reviewed in this article.
\end{abstract}

Keywords Posterior cortical atrophy $\cdot$ Alzheimer $\cdot$ Dementia . Ventral stream · Dorsal stream · MRI · Tractography · SPECT · PiB-PET · Presenilin 1 gene · Amyloid · Tau

\section{Introduction}

Posterior cortical atrophy (PCA) is a group of neurodegenerative disorders in which early complaints are predominantly visual. This syndrome was first described in 1988 when Benson et al. [1] reported five patients with prominent visual

This article is part of the Topical Collection on Neuro-Ophthalmology

F.-X. Borruat $(\bowtie)$

Department of Ophthalmology, University of Lausanne, Jules Gonin

Eye Hospital, Neuro-Ophthalmology Unit, Avenue de France 15,

1004 Lausanne, Switzerland

e-mail: francois.borruat@fa2.ch complaints and who all exhibited both Balint's and Gerstmann's syndromes. Owing to the presence of preponderant posterior atrophy of the brain on magnetic resonance imagin (MRI), they coined the term PCA [1]. Subsequent pathological studies have revealed Alzheimer's disease (AD) to be the most frequent cause of PCA, accounting for $80 \%$ of cases [2-4]. AD can present in patients older than 65 years (late-onset $\mathrm{AD}$ ) or in younger patients (early-onset $\mathrm{AD}$ ). Most frequently, AD manifests in patients older than 65 years with a prominent amnestic syndrome. On the contrary, patients with early-onset $\mathrm{AD}$ do not present prominent amnestic syndrome until late in their disease, but exhibit different prominent symptoms, such as limb apraxia, disturbances of either language or executive functions, or visuospatial functions (PCA) [5]. While the term "visual variant of AD" has been used popularly in the past, the more generic term PCA is presently favored because of both the heterogeneous etiologies that may present in such fashion and the fact that pathology confirmation is often lacking. As PCA results most frequently from $\mathrm{AD}$ pathology, the vast majority of recent literature on PCA is comparing PCA of presumed AD pathology to typical amnestic AD.

Since 1988, PCA has been gradually more frequently recognized and better characterized. PCA affects patients of both sexes, usually in their 50 s or early $60 \mathrm{~s}$. The onset is insidious, and patients with PCA frequently report symptoms that started years before diagnosis. Incidence and prevalence of PCA are unknown, but PCA accounts for $4 \%$ of dementia patients and is estimated to represent $5 \%$ of all $\mathrm{AD}$ presentations [6]. Initial complaints are mostly related to vision, reading, driving, and walking. Most of these symptoms can be suggestive of disturbances affecting either the afferent visual retino-calcarine or the efferent oculomotor pathways. In fact, the visual symptoms of PCA result from an impairment at a higher level of visual processing (beyond the occipital cortical V1, or Brodmann's area 17) and both the dorsal stream (occipito-parietal cortex, "where" pathway) and the ventral stream (occipito-temporal, "what" pathway) can be involved to various degrees [7, 8•]. Symptoms in PCA are then related to an impairment of the 
visuospatial and/or visuoperceptual performances. Neuropsychologic examination can disclose any of the following: alexia, Balint's syndrome (oculomotor apraxia, simultanagnosia, optic ataxia), Gerstmann's syndrome (agraphia, acalculia, finger anomia, right-left disorientation), prosopagnosia, or environmental agnosia.

The large majority of PCA articles relate to its most frequent etiology: Alzheimer's pathology. At an early stage, PCA patients differ from typical AD for the followings: memory disturbances are prominent in typical $\mathrm{AD}$, but absent or minimal in PCA; visual symptoms predominate in PCA; patient insight in PCA is usually preserved until late in the disease. Owing to the relative preservation of personal insight, some patients with PCA develop frank anxiety or depression, which can contribute to a delayed diagnosis. However, PCA is a progressive disorder and, over a few years, patients ultimately develop a more global dementia. Even if $\mathrm{AD}$ is the underlying pathology in more than $80 \%$ of cases, PCA remains a heterogenous group of diseases. Hence, despite the growing interest of researchers for PCA, research efforts are still hampered by the lack of definite clinical and paraclinical criteria for PCA. In 2012, the first meeting of the International Working Party on PCA was held in Vancouver, Canada, prior to the Alzheimer's Association International Conference, with a primary goal to define standardized clinical criteria for PCA [9]. Standardized diagnostic criteria and more homogenous and comparable cohorts of PCA patients might ultimately lead to the possibility of testing specific therapies for PCA.

\section{Clinical Manifestations of PCA}

Patients with PCA present with prominent visual symptoms, including difficulties in reading a text; problems with driving or parking a car; difficulties in identifying objects; walking difficulties in the presence of uneven ground, stairs, sidewalk borders, or escalators; and light sensitivity, sustained afterimages, or even visual hallucinations. Despite prominent visual symptoms, results from ophthalmological examinations are frequently unremarkable (or, at least, do not explain fully the clinical picture). Therefore, some patients will benefit from unnecessary procedures, such as cataract surgery.

Owing to the posterior localization of cerebral involvement in PCA, retrochiasmal visual pathways dysfunction can occur in PCA. Indeed, some cases of homonymous visual field defects were reported in the early literature on PCA, but both their prevalence and incidence was thought to be low. Recent reports tend to demonstrate that the incidence of homonymous defects in PCA is higher than previously thought, ranging from $50 \%$ to $100 \%[10-13]$. However, owing to the dementing nature of PCA, visual field examination can sometimes be challenging, and neither computerized static nor kinetic manual perimetry can be performed adequately by some patients.

Andrade et al. [14] reported visual hemineglect to be a frequent finding in PCA patients (16/24 patients). According to these authors, the line bisection test was more sensitive for detecting visual hemineglect in PCA compared to target cancellation tasks, and the presence of visual hemineglect did not correlate with disease duration or severity [14]. In another paper, the same group [15] reported a series of 15 PCA patients in whom visual left hemineglect (5/7 cases) was more frequent and more severe than right hemineglect ( $2 / 7$ cases). Neuroimaging results revealed the left hemineglect to be associated with the presence of both atrophy and hypoperfusion of the parieto-temporal and prefrontal regions [15]. In a later paper, Andrade et al. [16॰] further explored visual hemineglect in PCA, correlating visuospatial deficits and altered cerebral perfusion in a series of 27 patients. Visual hemineglect was found in 16 patients, with a surprisingly high incidence of right visual hemineglect (7/16 patients). They explained this high rate of right visual hemineglect by the presence of concurrent lesions of the right hemisphere [16 ${ }^{\bullet}$.

Basic visual processing and higher-order visual functions were assessed by Lehmann et al. [17•] in 21 PCA patients and correlated to cortical thickness. The basic visual skills were assessed with six tests: form detection, form coherence, form discrimination, color discrimination, motion coherence, and point localization. At least one result of basic visual function was impaired in all PCA patients, whereas $81 \%$ exhibited abnormal results for at least $3 / 6$ tests. Thus, no case of "pure" visual agnosia was found amongst 21 PCA patients. Patients with visuospatial defects (dorsal stream dysfunction) tended to exhibit lower occipito-parietal cortical thickness, whereas there was a trend towards lower occipito-temporal cortical thickness in patients with visuoperceptual defects (ventral stream dysfunction). However, despite these trends, neuroimaging per se did not support differentiation of PCA into dorsal versus ventral subtypes owing to the overlap of magnetic resonance imaging (MRI) findings between the two clinical groups [17•]. Amongst a group of 39 PCA patients, working memory deficits, simultanagnosia, and limb apraxia were found to be the most common abnormalities, occurring in $100 \%, 92 \%$, and $95 \%$, respectively, of the patients. A full Balint's or Gerstmann's syndrome was encountered in $31 \%$ and $36 \%$, respectively, of the patients [18•]. The combination of these two syndromes, as originally reported by Benson, must be very rare.

Optic ataxia is part of Balint's syndrome, a common disorder found in PCA. In optic ataxia from causes other than PCA, grasping performances were improved with eye closure. Quantitative measurements of visuomotor deficits were performed in four PCA patients [19॰]. Contrary to patients with optic ataxia from another cause, none of the PCA patients improved in reaching and grasping abilities with eye closure. 
Average speed was decreased upon eye closure, and, under visual guidance, all PCA patients produced highly curved reach trajectories when reaching either at midline or at the periphery. Both slowing the reaching movements and increasing the curvature of the reach trajectories might represent a compensation strategy developed as the consequence of decreased central parietal motor control [19•].

Reading difficulties are a frequent complaint in PCA, reported by about $80 \%$ of patients. Yong et al. [20] tested the reading performances of two PCA patients with prominent visual complaints. Despite a widespread dysfunction at the lower levels of visual processing (both patients failed all 10 tasks assessing early visual, visuoperceptual, and visuospatial processing) their reading performances were normal or near normal. The visual word form system is in the left fusiform gyrus, which was found to be intact in both patients. Some patients with PCA can retain normal reading despite widespread visual dysfunction [20].

Patients with PCA can complain of unusual symptoms, which can be puzzling to the physician. Crutch et al. [21] reported an interesting patient who complained of four symptoms, two of them previously reported in PCA (prolonged chromatic afterimages and reverse size effects) and two not previously reported in PCA (perceived motion of static stimuli and room tilt illusion). Reverse size effects (the ability to better read small than large prints) result from reduction in the "effective visual field" (i.e., attention disorder and eye movement disorders). Prolonged chromatic afterimages are thought to result from an excessive rebound inhibition of previously excited color selective neurons in V1 (primary visual cortex, occipital cortical area 17), as inhibitory interneurons in V1 are relatively spared in AD. Perceived motion of static stimuli was attributed to abnormal eye movements, namely unstable fixation. Room tilt illusion may result from lesions affecting the multimodal cells in the posterior parietal lobe, as they play an important role in integrating inputs from the visual, vestibular, and proprioceptive systems [21].

Picture agnosia is a specific lack of recognition of objects on pictures, whereas the real objects are either better or normally identified. While viewing a real object, binocular disparity cues can help its identification, whereas identification of an object from a two-dimensional photography requires preserved stereoscopic visual memory. Picture agnosia was found in 2/3 patients with PCA, but not in a group of seven typical amnestic AD patients serving as controls. The difference in the distribution of AD pathology between PCA and typical amnestic $\mathrm{AD}$ probably accounted for the absence of picture agnosia in typical amnestic AD patients. For some PCA patients, two-dimensional objects can be more difficult to identify than their three-dimensional counterparts [22].

Gardini et al. [23] reported a patient at an advanced stage of PCA. Neuropsychological examination disclosed two previously unreported deficits: defective visuospatial mental imagery and defective autobiographical memory. Anatomical and functional investigations with MRI and single-photon emission computerized tomography (SPECT) showed the former symptoms to be related to hypoperfusion in the parahippocampus and precuneus, whilst the later was associated with reduced blood flow in the parahippocampi [23].

Language impairment was initially reported by Benson et al. [1] in their seminal paper on PCA. However, until recently, very few reports have addressed language impairment in PCA. Magnin et al. [24] described a "logopenic syndrome" in 8/9 PCA patients, characterized by anomia, fluency impairment, and length-dependent deficit. The left parieto-temporal junction seems to play an important role in the "logopenic syndrome" of PCA patients [24]. Crutch et al. [25] examined the language profile both in PCA patients and in patients with the logopenic/phonological aphasia form of AD. Results were abnormal in both groups but PCA patients performed better than the logopenic/phonological aphasia patients. Detailed linguistic assessment might help to differentiate PCA due to AD from other causes of PCA [25].

Olfaction was found to be impaired similarly in patients with PCA and with typical amnestic AD. Further, around $30 \%$ of both PCA and AD patients exhibited a complete loss of olfaction. The high frequency of abnormal olfactory test results contrasted with the fact that olfactory symptoms were rarely reported by patients. The abnormal odor identification was associated with dysfunction of the right anteromedial temporal lobe dysfunction [26].

\section{Neuroimaging of PCA}

Since 1988, anatomical neuroimaging (mainly MRI) has been used to help diagnose PCA [1]. Occipitoparietal atrophy without mesiotemporal atrophy is classically described as the initial MRI findings of PCA. However, some cases exhibit either minimal or general cortical atrophy. When MRI does not support a diagnosis of PCA, but the patient's symptoms suggest PCA, functional neuroimaging can help the clinician to establish the diagnosis. In PCA, functional neuroimaging usually discloses selective posterior hypometabolism by fluorodeoxyglucose positron emission tomography (FDGPET) or hypoperfusion by SPECT. However, with the diagnosis of PCA being usually delayed, the first neuroimaging is performed at an already moderately advanced stage of PCA.

\section{MRI Studies}

The natural history of PCA on MRI was provided by Kennedy et al. [27•]. They reported one patient who volunteered as a healthy control, but complained of memory loss. Initial neuropsychological examination was normal, as was his MRI. Subsequently, over 5 years, both repeat MRI 
and clinical examinations revealed anomalies that progressively worsened over time. Cortical atrophy affected first the superior parietal and inferior temporal cortices. One year later, the inferior parietal and occipital areas were involved. Another year later, the medial temporal lobe was affected, and ultimately global posterior cerebral atrophy was present [27•].

At the early stage of the disease, MRI can separate PCA from typical AD: the former will show predominant posterior cortical atrophy, whereas the latter will show prominent atrophy of the medial temporal cortex. With an increased duration of the disease, it may become difficult to distinguish PCA from typical AD on MRI. Lehmann et al. [28•] reported serial imaging in 17 PCA patients, 16 typical AD patients, and 18 healthy controls. After 5 years of symptom duration, the MRI appearances of PCA and AD were alike: a widespread atrophy of grey matter. The rate of brain atrophy was five times higher in both PCA and typical AD when compared with healthy normals [28•]. Whereas MRI can differentiate PCA (occipitoparietal atrophy) from typical amnestic AD (mesiotemporal atrophy) at the early stage of the disease, it will not be able to do so at an advanced stage of neurodegeneration, the degree of grey matter atrophy being similar in AD and PCA.

Assessment of cortical atrophy on MRI can be done either via complex techniques such as volumetric analysis or via a visual subjective method, which is quicker. Koedam et al. [29] developed an MRI subjective visual rating scale for cortical atrophy (posterior, medial temporal lobe, and global atrophy), which they applied to a group of $60 \mathrm{AD}$ patients, comparing their results to 40 normal controls and 20 patients with other dementias. Intra-rater agreement was excellent, and the interrater agreement was good although more variable, as expected with a subjective method. Inter-rater agreement was best when assessing medial temporal lobe atrophy and was good, but lower, for posterior atrophy assessment. The visual rating method proposed by Koedam et al. [29] may be useful in a clinical setting for separating AD from other dementias. Lehmann et al. [30 ] concluded that posterior cerebral atrophy on MRI was a neuroimaging marker of $\mathrm{AD}$, even in the absence of medial temporal lobe atrophy. The finding of posterior cerebral atrophy on MRI may then help to distinguish $\mathrm{AD}$ from other dementias [30•]. However, the specificity of posterior atrophy on MRI for AD was challenged by O'Donovan et al. [31]. In a series of older patients, they found that posterior atrophy on MRI was not a reliable marker in older patients to distinguish AD from controls or to distinguish AD from dementia with Lewy bodies [31]. Cortical thickness was measured in 54 patients with PCA $(n=25)$, typical amnestic $\mathrm{AD}(\mathrm{n}=14)$, and also logopenic-progressive aphasia $(n=15)$, another distinctive presentation of AD. Temporo-parietal cortical thinning was common to all three forms of AD. Specific areas of atrophy in PCA were bilateral occipital and parietal regions. For the logopenic form of AD, the left temporo-parietal and superior temporal lobes were specifically thinned, whereas results for typical amnestic AD were distributed along a continuum between the previous two extremes [32•].

PCA is a neurodegenerative disorder, the diagnosis of which is suspected by clinical presentation and supported by results of neuropsychological tests and neuroimaging. The rare incidence of PCA precludes large prospective studies to be easily performed. Alves et al. [33] published the first metaanalysis of studies on brain morphometry in PCA. They collated 205 PCA patients (vs 184 typical AD and 249 healthy controls) from 11 published studies. The results of their analysis confirmed the previously published results: brain atrophy in PCA predominates in the right occipital gyrus and the right posterior lobe, whereas atrophy in typical $\mathrm{AD}$ predominates in the left hippocampus and parahippocampal gyrus [33].

Recent articles have hypothesized that cognitive functions might not depend solely on grey matter dysfunction, but could depend on the integrity of brain networks. Cognitive dysfunction might then result from interruption of information flow between different cortical areas. Using tractography, Migliaccio et al. [34] reported the findings of prominent white matter damage in the right hemisphere in addition to typical gray matter atrophy in one patient with PCA. This single case report supports the hypothesis of neural network dysfunction (white matter lesions) in neurodegenerative disorders. White matter atrophy of the ventral and dorsal streams have also been demonstrated by diffusion-tensor MRI in a series of seven PCA patients [35•]. Of note, all seven patients presented mostly symptoms derived from dysfunction of the ventral stream, whereas only $2 / 7$ exhibited symptoms derived also from dysfunction of the dorsal stream. All seven patients exhibited abnormalities in the ventral and fronto-occipital visual pathways (i.e., inferior longitudinal and inferior fronto-occipital fasciculi), whereas signal abnormalities in the dorsal visual pathways (fronto-parietal superior longitudinal fasciculus) were found only in the two patients with symptoms of visuospatial dysfunction. The results of this study suggest that Gerstmann's syndrome results from intraparietal white matter disconnection, another argument for the neural network dysfunction hypothesis [35•]. White matter atrophy can contribute to the clinical presentation of patients with PCA or other variants of $\mathrm{AD}$ versus typical $\mathrm{AD}$. White matter atrophy in PCA was more pronounced in the occipital lobe, whereas typical AD patients showed more damage in the medial temporal regions [36•].

\section{SPECT Studies}

Kas et al. [18•] studied a group of 39 PCA patients and correlated their cognitive deficits with the results of functional neuroimaging by SPECT [18•]. A group of 24 typical AD patients and 24 age-matched healthy controls was also studied. Posterior hypoperfusion was found in all PCA patients, and the extent of the hypoperfused areas progressively increased over 
time. Initially, hypoperfusion was found in the dorsal parietal cortices, with later involvement of the occipito-temporal cortices. When compared with typical AD patients, PCA was characterized by a more severe posterior hypoperfusion (occipital, parietal and posterior temporal cortices). Analysis of PCA patients by subgroups (symptom duration $\leq 2$ years, $\leq 3$ years, and $>3$ years) failed to reveal any specific hypoperfusion patterns [18•]. Further, they demonstrated also hypoperfusion of a small temporal area corresponding to the frontal eye field (FEF), as reported previously by Nestor et al. [37]. Involvement of FEF could play a role in the oculomotor apraxia present in some PCA patients [18•,37]. Another SPECT study in a series of 27 PCA patients revealed that visuospatial deficits, namely hemineglect, were associated with contralateral parietal cortex hypoperfusion and interhemispheric perfusion asymmetries $[16 \bullet]$.

\section{PET Studies}

In PCA, selective posterior hypometabolism can be demonstrated by FDG-PET, and FDG-PET has been used for several years to support a clinical suspicion of PCA. The pathology in the vast majority of patients with PCA ( $80 \%)$ is $\mathrm{AD}$, and cerebral amyloid deposits play a major role in $\mathrm{AD}$. In vivo imaging of cerebral amyloid deposits is now available with two different PET techniques: Pittsburgh compound B-PET (PiB-PET) and (18)F-florbetapir-PET. Investigation of PCA with these recent techniques has just begun and reports are few. Rosenbloom et al. [38•] reported the results of both PiBPET and FDG-PET in 12 PCA patients, 14 typical AD patients, and 30 normal controls. PiB-PET revealed diffuse deposits of amyloid in both PCA and typical AD patients, whereas glucose metabolism, as measured with FDG-PET, was found to be significantly decreased in the inferior occipitotemporal cortex of PCA patients. PiB-PET does not allow separation of PCA and $\mathrm{AD}$, at least in the mild-tomoderate stage of the disease. The authors concluded that both clinical features and hypometabolism of PCA were not related to fibrillary amyloid distribution. Individual susceptibility and vulnerability of specific cortical networks might determine the type of neurodegenerative pattern, that is, the specific presentation of AD [38•]. Similar results were reported by Cruz de Sousa et al. [39] in a series of nine PCA and nine matched AD patients. The authors found that PiB-PET abnormalities were similar between typical AD and PCA [39]. PiB-PET might not be helpful in differentiating typical AD from PCA.

PET using (18)F-florbetapir has not been applied yet to specifically study PCA. However, one study in AD patients revealed regional variations in the amount of amyloid deposits. Namely, prominent deposits of amyloid were found in the posterior association areas, and maximal deposition was in the frontal cortex [40].
Whereas FDG-PET remains a very helpful neuroimaging technique in the diagnosis of PCA, the implications of the newest techniques, PiB-PET and (18)F-florbetapir-PET, are not yet well defined.

\section{Pathology of PCA}

Etiologies of PCA include AD, dementia with Lewy bodies, subcortical gliosis, corticobasal degeneration, and prionassociated diseases. AD is the most frequent cause of PCA, accounting for about $80 \%$ of cases [2-4]. Recent pathology reports on PCA are scarce. Depaz et al. [41] reported on the occurrence of an early and longstanding dementia in five members of a family. Clinical and radiological presentations were typical of PCA. However, prion disease was suspected when one patient developed a subacute myoclonic encephalopathy with specific electroencephalography abnormalities, 16 years after disease onset. A mutation of the prion protein gene consisting of five octa repeats motifs located between R2 and R3 was found in the proband, as well as in other family members. Mutations in the prion gene protein can induce a dementing disorder resembling AD or PCA [41].

Several neurodegenerative diseases result from an abnormal accumulation of tau protein fragments within the central nervous system. Three- and four-repeat tauopathies have been associated with various clinical diagnoses. Jellinger et al. [42] reported a case of a patient with a post-mortem diagnosis of four-repeat tauopathy who clinically presented PCA with dysfunction of both ventral and dorsal streams [42]. CSF was atypical for $\mathrm{AD}$ (elevated t-tau, but normal $\mathrm{p}$-tau protein, and elevated $\beta$-amyloid 1-42; see the "Biology Studies" section). Post-mortem, a diagnosis of possible corticobasal degeneration was proposed in the total absence of cerebral amyloid deposits and due to the presence of tau-pathology [42].

One patient diagnosed early in childhood with Tourette's syndrome developed PCA in his 60s. The association might have been fortuitous, but it is notable that Tourette's syndrome has been reported to occur with other form of dementias [43].

\section{Genetics of PCA}

The genetics of PCA are still elusive. Mutations in presenilin 1 gene (PSEN1) are commonly found in familial early-onset $\mathrm{AD}$, and result in a reduction of $\gamma$-synthetase activity - an enzyme involved in amyloid metabolism. One patient with PCA was recently reported to harbor a novel genetic mutation, at codon 211 of the PSEN1 gene. Results from an in silico (computer-based) analysis of the mutation lead the authors to conclude that the novel PSEN1 I211M mutation could be responsible for the PCA phenotype [44]. 


\section{Biology Studies: CSF and Serum Abnormalities in PCA}

There is a major interest in being able to diagnose PCA in vivo with some certainty. The CSF biomarkers of typical AD have been defined as a combination of elevated total-tau ( $\mathrm{t}$-tau) and phosphorylated-tau ( $\mathrm{p}$-tau) proteins, and decreased amyloid- $\beta$ 42 (A $\beta 42$ ). When these three biomarkers are abnormal, AD neuropathology is predicted with both a high sensitivity (90\%) and specificity (85\%). Seguin et al. [45•] assessed the cerebrospinal fluid (CSF) biomarkers in a series of 22 patients with PCA, and $77 \%$ of cases (17/22) showed CSF profiles identical to typical AD. Three patients showed CSF profiles that were atypical for AD (either elevated t-tau and $\mathrm{p}$ tau, or decreased $A \beta 42$ ). Only $2 / 22$ patients exhibited a normal CSF profile, and these patients belonged to a subgroup of three clinically defined PCA-corticobasal syndrome patients. CSF can be useful to determine in vivo which etiology is underlying PCA [45•]. Similar results were obtained from Cruz de Sousa et al. [46] who reported the same CSF profile in nine patients with PCA and nine matched typical AD patients. Both PCA and typical AD patients exhibited elevated t-tau and p-tau proteins levels, and decreased A $\beta 42$ level. Cruz de Sousa et al. [47] refined further the analyses of CSF biomarkers. The t-tau/A $\beta 42$ and $p$-tau/A $\beta 42$ ratios allowed to differentiate with both high sensitivity and specificity typical AD patients from other causes of cortical dementias, namely semantic and frontotemporal dementias. However, these biomarkers were not useful in differentiating typical AD from its variants, PCA and logopenic AD [47]. Beaufils et al. [48] refined the analysis of CSF by introducing the ratio of $A \beta 42 /$ $\mathrm{A} \beta 40$, which was decreased in all PCA $(n=22)$ and typical $\mathrm{AD}(\mathrm{n}=22)$ patients of their series. In the presence of an atypical CSF profile for $A D$, the use of the $A \beta 42 / A \beta 40$ ratio proved useful in establishing the diagnosis of AD pathology, as demonstrated in 4/22 PCA patients who exhibited an otherwise atypical CSF profile (t-tau and/or p-tau not elevated) [48].

Anti-amyloid- $\beta$ antibodies are found in the serum of normal elderly individuals, and their role in the pathogenesis of $\mathrm{AD}$ is not clear. In one study of 13 typical AD and eight PCA patients, Dorothée et al. [49•] reported a specific decrease in the level of anti-amyloid- $\beta$ antibodies in the serum of the PCA patients only. Further, the serum level of IgG1 was significantly more lowered than for IgG3. As IgG1 and IgG3 are both involved in neuroprotection and neuroinflammation, this specific serologic pattern might have significance in the pathogenesis of PCA [49•].

\section{Treatment of PCA}

Unfortunately, very little has been published on therapeutic options in PCA. Patients with PCA are usually treated with cholinesterase inhibitors, by analogy with treatment of typical AD. However, there is no evidence-based medicine for the efficacy of cholinesterase inhibitors in PCA. A review on the pharmacologic therapies of typical AD emphasized that, since 2003, no new drug was marketed for AD. Today, cholinesterase inhibitors and memantine are the only options for typical AD. Future therapies might be directed at controlling the levels of amyloid deposits, decreasing tau aggregates, or modulating neurotransmitters [50•]. McMonagle [51] reported his experience in treating 10 PCA patients with donepezil in an unblinded and pragmatic study. Global cognitive scores were significantly improved, but no improvement was detectable for the visuospatial scores.

Non-pharmacologic therapies have been proposed for a few PCA patients. A psycho-educative program was offered to four PCA patients and their caregivers [52]. A team consisting of a neurologist, psychologist, nurse, and ergotherapist animated the sessions. Both patients and caregivers benefited from this approach by improving their overall knowledge of PCA. Further, the anxiety of the caregivers decreased significantly. However the quality of life of the PCA patients did not improve [52]. A multidisciplinary

Table 1 Diagnosis of posterior cortical atrophy

Core features

- Insidious onset and gradual progression

- Prominent and disabling visual complaints

- No ocular disease explaining the symptoms

- Visuoperceptual and visuospatial impairment

- Relatively preserved anterograde memory and insight

- Any of the following:

- Balint's syndrome (partial or complete)

- Gerstmann's syndrome (partial or complete)

○ Homonymous visual field defect

$\circ$ Environmental disorientation

Supportive features

- Alexia

- Prosopagnosia

- Prolonged chromatic after-images

- Dressing or ideomotor apraxia

- Presenile onset

Investigations

- Neuropsychological assessment

- Structural neuroimaging (magnetic resonance imaging/ computed tomography)

$\circ$ Absence of tumor or stroke

- Evidence of posterior atrophy (occipital, parietal and temporal areas)

- Functional neuroimaging (positron emission tomography/ single-photon emission computerizd tomography)

○ Posterior hypometabolism/hypoperfusion 
approach combining speech therapy, occupational therapy, and physiotherapy was applied to a single PCA patient. Marked improvement of the patient's autonomy was observed after 6 months of therapy, thanks to a better understanding of PCA and to the development of compensation strategies for daily life activities (e.g., dressing, reading) [53].

\section{Conclusions}

PCA is a progressive neurodegenerative heterogeneous disorder with a poor prognosis, as an evolution towards global dementia is frequent. PCA is suspected when complex unexplained visual symptoms are prominent in a patient usually in her/his 50s. Diagnosis will be supported by formal neuropsychological examination and neuroimaging (Table 1). The majority of PCA cases results from AD pathology, and results from recent neuroimaging and biological studies in PCA confirm that association in vivo. Significant improvement in clinical diagnosis, neuroimaging and biological studies have recently occurred and help to distinguish PCA from other dementing conditions. Unfortunately, there is still no specific treatment for PCA, and this is owing to both the rarity and the heterogeneity of the disorder. However, the specific interests of research teams for PCA and the recent launch of the International Working Party on PCA [8•] will certainly contribute to improve both our knowledge of PCA and the management of PCA patients in a near future.

\section{Compliance with Ethics Guidelines}

Conflict of Interest François-Xavier Borruat declares that he has no conflict of interest.

Human and Animal Rights and Informed Consent This article does not contain any studies with human or animal subjects performed by any of the authors.

\section{References}

Papers of particular interest, published recently, have been highlighted as:

- Of importance

1. Benson DF, Davis RJ, Snyder BD. Posterior cortical atrophy. Arch Neurol. 1988;45:789-93.

2. Victoroff J, Ross GW, Benson DF, et al. Posterior cortical atrophy: neuropathologic correlations. Arch Neurol. 1994;51:269-74.

3. Tang-Wai DF, Graff-Radford NR, Boeve BF, et al. Clinical, genetic, and neuropathologic characteristics of posterior cortical atrophy. Neurology. 2004;63:1168-74.

4. Renner JA, Burns JM, Hou CE, et al. Progressive posterior cortical dysfunction. A clinicopathologic series. Neurology. 2004;63:1175-80.
5. Mendez MF, Lee AS, Joshi A, Shapira JS. Nonamnestic presentations od early-onset Alzheimer's disease. Am J Alzheimers Dis Other Dement. 2012;27:413-20.

6. Snowden JS, Stopford CL, Julien CL, et al. Cognitive phenotypes in Alzheimer's disease and genetic risk. Cortex. 2007;43:835-45.

7. Untergleider LG, Haxby JV. "What" and "where" in the human brain. Curr Opin Neurol. 1994;4:157-65.

8. - Crutch JS, Lehmann M, Schott JM, et al. Posterior cortical atrophy. Lancet Neurol. 2012;11:170-8. A very clear and concise review of $P C A$, written by world experts in the field of PCA.

9. Crutch SJ, Schott JM, Rabinovici GD, et al. Shining a light on posterior cortical atrophy. Alzheimers Dement. 2013;9:463-5.

10. Lee AG, Martin CO. Neuro-ophthalmic findings in the visual variant of Alzheimer's disease. Ophthalmology. 2004;111:376-81.

11. Formaglio M, Krolak-Salmon P, Tilikete C, et al. Homonymous hemianopia and posterior cortical atrophy. Rev Neurol. 2009;165: 256-62.

12. Pelak VS, Smyth SF, Boyer PJ, Filley CM. Computerized visual field defects in posterior cortical atrophy. Neurology. 2011;77:2119-22.

13. René R, Munoz S, Campdelacreu J, et al. Complex visual manifestations of posterior cortical atrophy. J Neuroophthalmol. 2012;32: 307-12.

14. Andrade K, Samri D, Sarazin M, et al. Visual neglect in posterior cortical atrophy. BMC Neurol. 2010;10:68.

15. Andrade K, Kas A, Valabrègue R, et al. Visuospatial deficits in posterior cortical atrophy: structural and functional correlates. J Neurol Neurosurg Psychiatry. 2012;83:860-3.

16. - Andrade K, Kas A, Samri D, et al. Visuospatial deficits and hemispheric perfusion asymmetries in posterior cortical atrophy. Cortex. 2013;49:940-7. Case-control study of hemineglect and brain perfusion in a large group of $P C A$ patients.

17. • Lehmann M, Barnes J, Ridgway GR, et al. Basic visual function and cortical thickness patterns in posterior cortical atrophy. Cereb Cortex. 2011;21:2122-32. This is one of the few studies which has addressed basic visual deficits in a large series of PCA patients. Regional cortical thickness analysis per se did not allow to separate patients with specific visuospatial from those with visuoperceptual disorders.

18. - Kas A, Cruz de Souza L, Samri D, et al. Neural correlates of cognitive impairment in posterior cortical atrophy. Brain. 2011;134: 1464-78. Large study correlating cognitive impairment and brain perfusion deficits by SPECT.

19. - Meek BP, Shelton P, Marotta JJ. Posterior cortical atrophy: visuomotor deficits in reaching and grasping. Front Hum Neurosci. 2013;7:1-16. First quantitative study of visuomotor deficits in PCA.

20. Yong KXX, Warren JD, Warrington EK, Crutch SJ. Intact reading in patients with profound early visual dysfunction. Cortex. 2013 February 13 [Epub ahead of print].

21. Crutch SJ, Lehmann M, Gorgoraptis N, et al. Abnormal visual phenomena in posterior cortical atrophy. Neurocase. 2011;17:160-77.

22. Sugimoto A, Midorikawa A, Koyama S, et al. Picture agnosia as a characteristic of posterior cortical atrophy. Eur Neurol. 2012;68: 34-41.

23. Gardini S, Concari L, Pagliara S, et al. Visuo-spatial imagery impairment in posterior cortical atrophy: a cognitive and SPECT study. Behav Neurol. 2011;24:123-32.

24. Magnin E, Sylvestre G, Lenoir F, et al. Logopenic syndrome in posterior cortical atrophy. J Neurol. 2013;260:528-33.

25. Crutch SJ, Lehmann M, Warren DJ, Rohrer JD. The language profile of posterior cortical atrophy. J Neurol Neurosurg Psychiatry. 2013;84: 460-6.

26. Witoonpanich P, Cash DM, Shakespare TJ, et al. Olfactory impairment in posterior cortical atrophy. J Neurol Neurosurg Psychiatry. 2013;84:588-90.

27. Kennedy J, Lehmann M, Sokolska MJ, et al. Visualizing the emergence of posterior cortical atrophy. Neurocase. 2012;18:248-57. A 
case report describing the very early MRI deficits of PCA and its evolution over the years.

28. - Lehmann M, Barnes J, Ridgway GR, et al. Global grey matter changes in posterior cortical atrophy: a serial imaging study. Alzheimers Dement. 2012;8:502-12. This case-control MRI study demonstrated that PCA evolves from a focal to a global pattern of gray matter atrophy over time.

29. Koedam ELGE, Lehmann M, van der Flier WM, et al. Visual assessment of posterior atrophy development of a MRI rating scale. Eur Radiol. 2011;21:2618-25.

30. • Lehmann M, Koedam ELGE, Barnes J, et al. Posterior cerebral atrophy in the absence of medial temporal lobe atrophy in pathologically-confirmed Alzheimer's disease. Neurobiol Aging. 2012;33:627-e1-627.e12. This large MRI study questioned whether posterior atrophy was a marker of $A D$, and the authors found so.

31. O'Donovan J, Watson R, Colloby S, et al. Does posterior cortical atrophy on MRI discriminate between Alzheimer's disease, dementia with Lewy bodies, and normal aging? Int Psychogeriatr. 2013;25: $111-9$.

32. - Ridgway GR, Lehmann M, Barnes J, et al. Early-onset Alzheiner disease clinical variants. Multivariate analyses of cortical thickness. Neurology. 2012;79:80-4. This large case-control MRI study demonstrated that the clinical variants of $A D$, including $P C A$, fall within the spectrum of the neuroanatomical defects of $A D$.

33. Alves J, Soares JM, Sampaio A, Goncalves OF. Posterior cortical atrophy and Alzheimer's disease: a meta-analytic review of neuropsychological and brain morphometry studies. Brain Imaging Behav. 2013 May 21 [Epub ahead of print].

34. Migliaccio R, Agosta F, Toba MN, et al. Brain networks in posterior cortical atrophy: a single case tractography study and literature review. Cortex. 2012;48:1298-309.

35. - Migliaccio R, Agosta F, Scola E, et al. Ventral and dorsal visual streams in posterior cortical atrophy: a DT MRI study. Neurobiol Aging. 2012;33:2572-84. A case series study that investigated the topography of specific white matter tract lesions in PCA, and correlated it with clinical defects of either the dorsal or the ventral stream.

36. - Migliaccio R, Agosta F, Possin KL, et al. White matter atrophy in Alzheimer's disease variants. Alzheimers Dement. 2012;8:S78-87. A large case-controlled MRI series investigated whether specific patterns of white matter atrophy were present in PCA and other variants of AD. Results support the hypothesis that cognitive deficits in PCA result from a neural network dysfunction involving both gray and white matter.

37. Nestor PJ, Caine D, Fryer TD, et al. The topography of metabolic deficits in posterior cortical atrophy (the visual variant of Alzheimer's disease) with FDG-PET. J Neurol Neurosurg Psychiatry. 2003;74: 1521-9.

38. - Rosenbloom MH, Alkalay A, Agarwal N, et al. Distinct clinical and metabolic deficits in PCA and AD are not related to amyloid distribution. Neurology. 2011;6:1789-96. This case-controlled study investigated the relationship of amyloid deposits and clinical/metabolic defects in PCA and AD. Diffuse amyloid deposits were found in $A D$ and PCA, and did not correlate with cortical dysfunction in PCA, whereas posterior glucose hypometabolism differentiated PCA from $A D$.

39. Cruz de Sousa L, Corlier F, Habert MO, et al. Similar amyloid- $\beta$ burden in posterior cortical atrophy and Alzheimer's disease. Brain. 2011;134:2036-43.

40. La Joie R, Perrotin A, Barré L, et al. Region-specific hierarchy between atrophy, hypometabolism, and $\beta$-amyloid $(\mathrm{A} \beta)$ load in Alzheimer's disease dementia. J Neurosci. 2012;32:16265-73.

41. Depaz R, Haik S, Peoc'h K, et al. Long-standing prion dementia manifesting as posterior cortical atrophy. Alzheimer Dis Assoc Disord. 2012;26:289-92.

42. Jellinger KA, Grazer A, Petrovic K, et al. Four-repeat tauopathy clinically presenting as posterior cortical atrophy: atypical corticobasal degeneration? Acta Neuropathol. 2011;121:267-77.

43. Fragassi NA, Chiacchio L, Errichiello L, et al. Posterior cortical atrophy with prominent alexia without agraphia in a Tourette syndrome. Neurol Sci. 2011;32:1129-33.

44. Sitek EJ, Narozanska E, Peplonska B, et al. A patient with posterior cortical atrophy possesse a novel mutation in the presenilin 1 gene. PLoS ONE. 2013;8:e61074.

45. - Seguin J, Formaglio M, Perret-Liaudet M, et al. CSF biomarkers in posterior cortical atrophy. Neurology. 2011;76:1782-8. Very useful results from this large prospective study of CSF profile of $P C A$ versus $A D$.

46. Cruz de Sousa L, Corlier F, Habert MO, et al. Similar amyloid- $\beta$ burden in posterior cortical atrophy and Alzheimer's disease. Brain. 2011;134:1036-43.

47. Cruz de Sousa L, Lamari F, Belliard S, et al. Cerebrospinal fluid biomarkers in the differential diagnosis of Alzheimer's disease from other cortical dementias. J Neurol Neurosurg Psychiatry. 2011;82: $240-6$.

48. Beaufils E, Dufour-Rainfray D, Hommet C, et al. Confirmation of the amyloidogenic process in posterior cortical atrophy: value of the A $\beta 42 / A \beta 40$ ratio. J Alzheimers Dis. 2013;33:775-80.

49. - Dorothée G, Bottlaender M, Moukari E, et al. Distinct patterns of antiamyloid- $\beta$ antibodies in typical and atypical Alzheimer disease. Arch Neurol. 2012;69:1181-5. Studying the patterns of antiamyloid$\beta$ antibodies might help a better categorization of patients suspected of PCA.

50. - Schneider LS. Alzheimer disease pharmacologic treatment and treatment research. Continuum (Minneap Minn). 2013;19:339-57. Although not directed specifically at PCA, this paper extensively reviewed both the actual and the future modalities to treat $A D$.

51. McMonagle P. Posterior cortical atrophy and cholinesterase inhibitors: do they help? Neurology. 2010;74:A410.

52. Videaud H, Torny F, Cartz-Piver L, et al. Impact of drug-free care in posterior cortical atrophy: preliminary experience with a psychoeducative program. Rev Neurol. 2012;168:861-7.

53. Weill-Chounlamountry A, Poncet F, Crop S, et al. Physical medicine and rehabilitation multidisciplinary approach in a case of posterior cortical atrophy. Ann Phys Rehab Med. 2012;55:430-9. 